\title{
A Review of Internet of Things in Healthcare
}

\author{
Md. Khashrul Alam \\ Professor, Business Administration Discipline, Khulna University, Khulna, Bangladesh
}

\begin{abstract}
Internet of Things is a dynamic global network infrastructure with self configuring capabilities based on standard and interoperable communication protocols where physical and virtual 'Things' have identities, physical attributes, and virtual personalities and use intelligent interfaces, and are seamlessly integrated into the information network. Internet of Things infrastructure is letting devices to collect, record, analyze data that was not accessible before. The Internet of Things can play a significant role in managing the chronic diseases such as asthma, cardiac related diseases, stroke, hypertension etc. remotely. The study is based on secondary data sources. It shows the application and challenges of IoT in healthcare.
\end{abstract}

Keywords: Radio Frequency Identification, Wireless sensor networks, body area network, Trustworthiness.

\section{INTRODUCTION}

Health care is a fundamental right of every citizen. Government is committed to provide better health care to each and every person of the country. In reality, health care system of many countries are plagued by corruption, poor management, lack of skilled health care term to illustrate the power of connecting Radioprofessionals, lack of proper monitoring and other Frequency Identification (RFID) tags used in corporate challenges. As a result, getting better health care is almost supply chains to the Internet in order to count and track a dream for the patients. Internet of Things (IoT) goods without the need for human intervention. The term technologies like wireless sensor networks, radio IoT was initially proposed to refer to uniquely identifiable frequency identification (RFID), Bluetooth, ZigBee can be interoperable connected objects with radio-frequency used to facilitate better health care. By embedding IoTenabled devices in medical equipment, healthcare professionals will be able to monitor patients more effectively - and use the data gleaned from the devices to figure out who needs the most hands-on attention. In other words, by making the most of this network of devices, healthcare professionals could use data to create a system of proactive management - as they say, prevention is better than the cure [18].
IoT technologies include many other technologies and devices such as barcodes, smart phones, social networks, and cloud computing are being used to form an extensive network for supporting IoT which are shown in the following diagram [5]-[8].
The term "Internet of Things" (IoT) was first used in 1999 by British technology pioneer Kevin Ashton to describe a system in which objects in the physical world could be identification (RFID) technology [1].

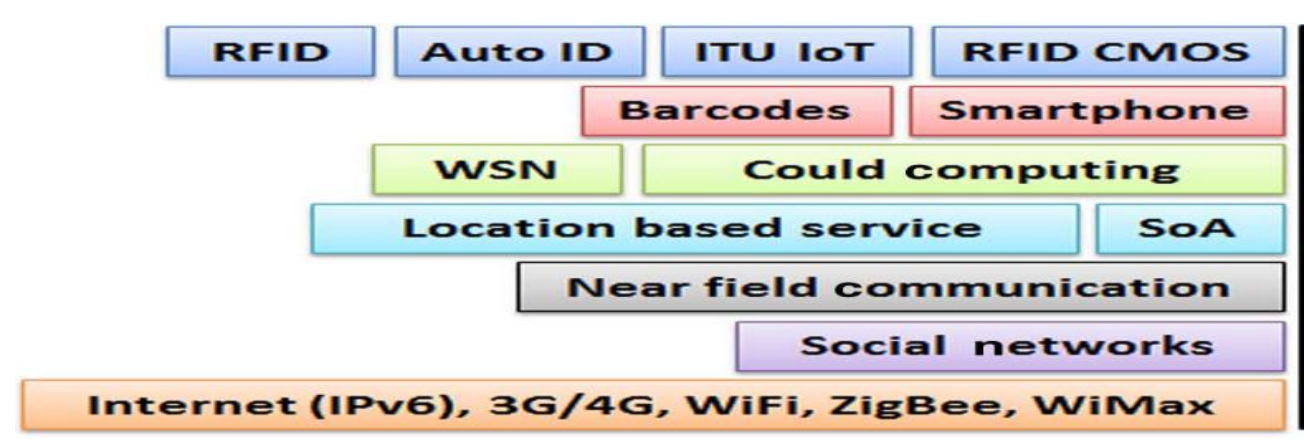

Fig.1. Technologies associated with IoT.

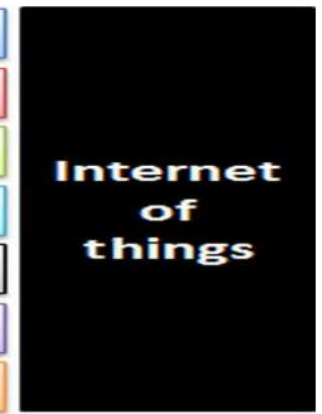


physical environment. Each device is capable of Their data rates are limited by the applications. WMSNs, monitoring-sensing-and/or

displaying-actuatinginformation. A sensor node is capable of gathering sensory information, processing it in some manner, and communicating with other nodes in the network [10]. When wireless sensor networks are designed for medical applications they are often referred to as wireless medical sensor networks (WMSNs). Wireless medical sensor networks have delivered significant improvements to the healthcare industry in the 21 st century. Wireless medical sensors are arranged on a patient's body and can be used to closely monitor the physiological condition of patients. These medical sensors monitor the patient's vital body signs (e.g temperature, heart rate, blood pressure, oxygen saturation, etc.) and transmit the data in a timely fashion to some remote location without human intervention. A doctor can interpret these sensor readings to assess a patient's condition. Thus patients could benefit from continuous long-term monitoring after being discharged from the hospital6. WMSNs will continue to play a central role in the future of modern healthcare as continuous and ubiquitous monitoring becomes increasingly important in order to shorten the amount of interaction needed between physicians and their patients to facilitate reduction of recently coined to unite researchers to form interdisciplinary teams with expertise in bioengineering, electronics, computer science and engineering, medicine, among others. Wireless medical sensor networks differ from traditional wireless sensor networks (WSNs). Traditional WSNs are independent and automatic, utilized in a large scale in either a fixed or distributed manner. however, involve direct human involvement (i.e., patient, doctor, nurse, other providers, etc.), are utilized in a small scale (i.e., depending on usability), must support mobility in order for the patient to be able to carry the device, and demand high data rates, with reliable communication and multiple recipients. WMSNs carry the promise of qualityof-care across wide variety of healthcare applications as they can be wearable, implantable, portable, and integrated on many types of wireless communication motes[11].

Wireless sensor networks have emerged as a feasible technology for a myriad of applications, including many different health care applications. WSN technology can be adapted for the design of practical Health Care WSNs (HCWSNs) that support the key system architecture requirements of reliable communication, node mobility support, multicast technology, energy efficiency, and the timely delivery of data[12].The application of the Wireless Sensor Networks in healthcare systems can be divided into three categories:

1. Monitoring of patients in clinical settings

2. Home \& elderly care centre monitoring for chronic and elderly patients

3. Collection of long-term databases of clinical data

The overview of a simple wireless sensor network application scenario is depicted in Fig. 2 . Based on this observation, in a typical scenario, there are four different categories of actors other than the power users of the systems such as administrators and developers. They are children, elderly and chronically ill, car giver and health care professionals.

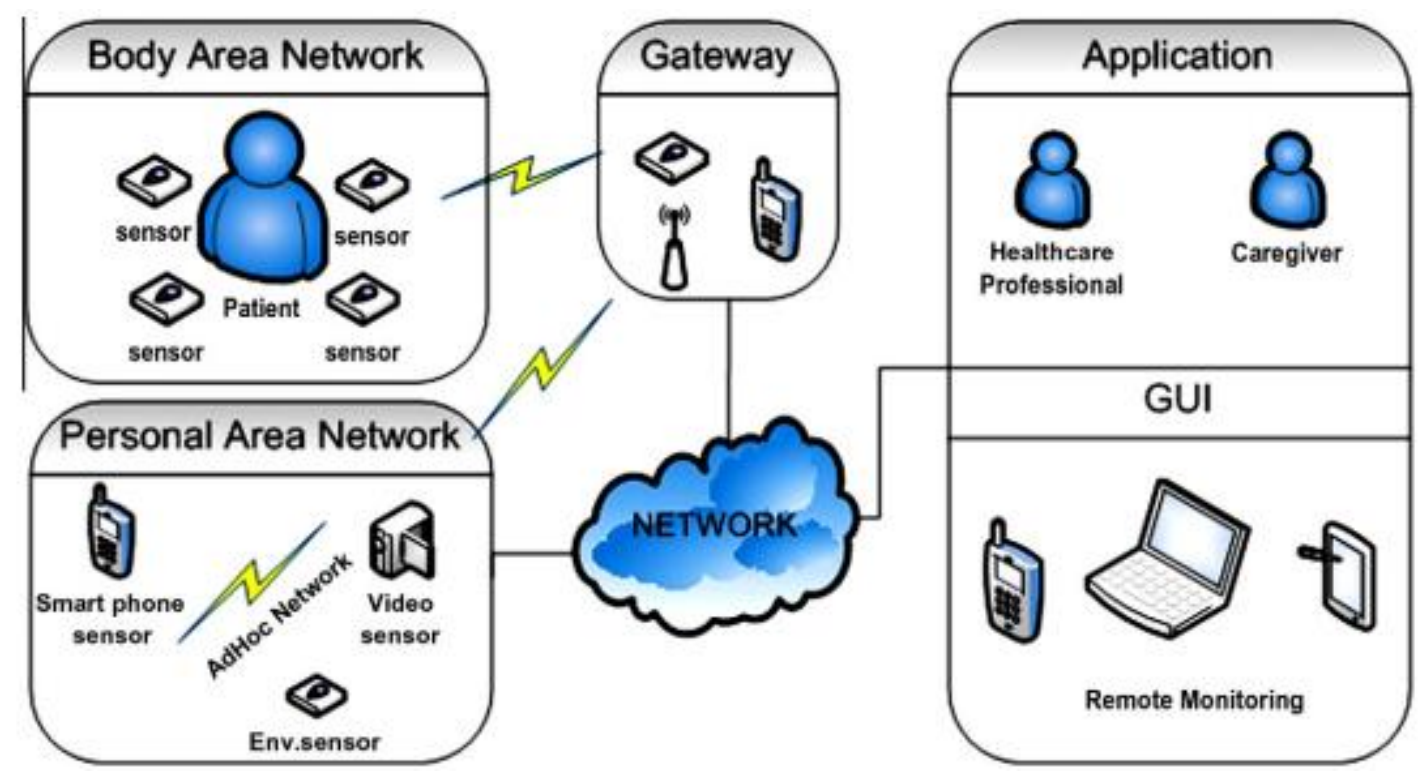

Fig.2. Overview of a simple WSN application scenario for healthcare.

These groups of actors constantly interact with the Area Network Subsystem, (iii) Gateway to the Wide Area wireless sensor network healthcare systems by using Networks, (iv) Wide Area Networks, and (v) End-user different subsystems. There are five subsystems in such a healthcare monitoring application. In Table1, the design scenario: (i) Body Area Network Subsystem, (ii) Personal considerations of these subsystems are provided 
The IoT based healthcare application faces many novel challenges, such as, reliable data transmission, node mobility support and fast event detection, timely delivery of data, power management, node computation and middleware. Further however, deploying new technologies in healthcare applications without considering security often makes patient privacy vulnerable. For instance unauthorized collection and use of patient data by potential adversaries (such as insurance agents, for political reasons, rival coaches, personal enemies etc.) can cause life-threatening risks to the patient, or make the patient's private matters publically available.

For example, in a simple scenario, a patient's body sensors transmit his/her body data to a nurse/caregiver; it may happen that an attacker is also eaves dropping the patient data while the data is transmitting, and consequently the patient's privacy is breached. In worst case scenario the attacker can upload the patient data on s social web site (FaceBook or Twitter, etc.), and thus pose risks to the patient's privacy. There is no doubt that IoT based healthcare can offer many advantages to patient monitoring, but the physiological data of an individual are highly vulnerable, so security and privacy become some of the big concerns for healthcare applications, especially when it comes to adopting wireless technology

Other important challenges can be the reliability and trustworthiness of the data collected through IoT technologies. Healthcare applications impose strict requirements on end-to-end system reliability and data delivery. For example, pulse oximetry applications, which measure the levels of oxygen in a person's blood, must deliver at least one measurement every 30 seconds. A number of factors complicate the systems' ability to provide the trustworthiness that applications require. First, medical facilities, where some of these systems will be deployed, can be very harsh environments for radio frequency (RF) communications. The application should be reliable. In other words health care sector has to manage three issues regarding reliability [19]. As for example the issues of reliability are: reliable data measurement, reliable data communication, and reliable data analysis.

\section{CONCLUSION}

IoT is an emerging technology for many sectors. Healthcare sector can get tremendous benefits through these technologies. Particularly critical patients as well as patients suffering from chronical diseases i.e asthma, cardiac and stroke related can get help in an unprecedented way. As for example patients would be able to get prompt treatment or caregiver support irrespective of where the patients are staying or living. In this paper technology related with IoT and framework for IoT enabled healthcare are presented briefly. At the end of the paper some of the challenges of IoT based healthcare are shown.

\section{REFERENCES}

[1] R. van Kranenburg, (2007) "The Internet of Things: A Critique of Ambient Technology and the All-Seeing Network of RFID." Amsterdam, The Netherlands: Institute of Network Cultures.

[2] R. van Kranenburg, E. Anzelmo, A. Bassi, D. Caprio, S. Dodson, and M. Ratto, (2011) "The internet of things," in Proc. 1st Berlin Symp. Internet Soc., Berlin, Germany, pp. 25-27.

[3] Sunghae Jun, (2014) "Technology Analysis For Internet of Things Using Big Data Learning", International Journal of Research in Engineering and Technology, Volume: 03 Issue: 12.

[4] D. Uckelmann, M. Harrison, and F. Michahelles,(2011) "An architectural approach towards the future internet of things," in Architecting the Internet of Things. D. Uckelmann, M. Harrison, and F. Michahelles, Eds., New York, NY, USA: Springer, pp 1-24. 
[5] S. Li, L. Xu, X. Wang, and J. Wang,(2012) "Integration of hybrid wireless networks in cloud services oriented enterprise information systems," Enterp. Inf. Syst., vol. 6, no. 2, pp. 165-187.

[6] Q. Li, Z. Wang, W. Li, J. Li, C. Wang, and R. Du,(2013) "Applications integration in a hybrid cloud computing environment: Modelling and platform,” Enterp. Inf. Syst., vol. 7, no. 3, pp. 237271.

[7] Li Da Xu, Senior Member, IEEE, Wu He, and Shancang Li.(2014) "Internet of Things in Industries: A Survey" IEEE Transactions On Industrial Informatics, Vol. 10, No. 4,

[8] L. Atzori, A. Iera, and G. Morabito, (2010) "The Internet of Things: A survey," Computer Networks, Vol. 54, Iss. 15, pp. 2787-2805. Stroulia, Eleni, et al,(2009) "Software Engineering for Health Education and Care Delivery Systems: The Smart Condo Project", IEEE SEHC .

[9] Li, Yingshu, My Thai, and Weili Wu,(2010) "Wireless Sensor Networks and Applications", Springer.

[10] Kumar, Pardeep, etall., (2012) "E-SAP: Efficient-Strong Authentication Protocol for Healthcare Applications Using Wireless Medical Sensor Networks, Sensors, 1625 - 1647.

[11] Suryadevara, N. K.,(2011) "Wireless Sensor Network Based Safe Home to Care Elderly People: ARealistic Approach", Recent Advances in Intelligent Computational Systems (RAICS), IEEE.

[12] KIPRIS (2014) Korea Intellectual Property Rights Information Service, http://www.kipris.or.kr.

[13] IEEE (2014) IEEE xplore, http://ieeexplore.ieee.org.

[14] USPTO (2014) The United States Patent and Trademark Office, http://www.uspto.gov.

[15] K. Ashton. (2009) .Internet of things. RFID J. Available http://www.rfidjournal.com/articles/view?4986

[16] www.internetsociety.org

[17] http://www.computerworlduk.com/iot/iot-centred-healthcaresystem-3643726/

[18] https://datafloq.com/read/internet-of-things-revolutionizehealthcare/1069 